\title{
Photobiomodulation with near infrared light mitigates Alzheimer's disease-related pathology in cerebral cortex - evidence from two transgenic mouse models
}

Sivaraman Purushothuman ${ }^{1,2}$, Daniel M Johnstone ${ }^{1,2^{*}}$, Charith Nandasena ${ }^{1,2}$, John Mitrofanis ${ }^{1,3}$ and Jonathan Stone $e^{1,2}$

\begin{abstract}
Introduction: Previous work has demonstrated the efficacy of irradiating tissue with red to infrared light in mitigating cerebral pathology and degeneration in animal models of stroke, traumatic brain injury, parkinsonism and Alzheimer's disease (AD). Using mouse models, we explored the neuroprotective effect of near infrared light (NIr) treatment, delivered at an age when substantial pathology is already present in the cerebral cortex.

Methods: We studied two mouse models with AD-related pathologies: the K369l tau transgenic model (K3), engineered to develop neurofibrillary tangles, and the APPswe/PSEN1dE9 transgenic model (APP/PS1), engineered to develop amyloid plaques. Mice were treated with NIr 20 times over a four-week period and histochemistry was used to quantify AD-related pathological hallmarks and other markers of cell damage in the neocortex and hippocampus.

Results: In the K3 mice, NIr treatment was associated with a reduction in hyperphosphorylated tau, neurofibrillary tangles and oxidative stress markers (4-hydroxynonenal and 8-hydroxy-2'-deoxyguanosine) to near wildtype levels in the neocortex and hippocampus, and with a restoration of expression of the mitochondrial marker cytochrome $c$ oxidase in surviving neurons. In the APP/PS1 mice, Nir treatment was associated with a reduction in the size and number of amyloid- $\beta$ plaques in the neocortex and hippocampus.
\end{abstract}

Conclusions: Our results, in two transgenic mouse models, suggest that Nlr may have potential as an effective, minimally-invasive intervention for mitigating, and even reversing, progressive cerebral degenerations.

\section{Introduction}

Alzheimer's disease $(\mathrm{AD})$ is a chronic, debilitating neurodegenerative disease with limited therapeutic options; at present there are no treatments that prevent the physical deterioration of the brain and the consequent cognitive deficits. Histopathologically, AD is characterised by neurofibrillary tangles (NFTs) of hyperphosphorylated tau protein and amyloid-beta $(A \beta)$ plaques $[1,2]$. The extent of these histopathological features is considered to vary with and to determine clinical disease severity [2]. While the

\footnotetext{
* Correspondence: Daniel.Johnstone@sydney.edu.au

'Bosch Institute, University of Sydney, NSW 2006, Australia

2Discipline of Physiology, Anderson Stuart Building F13, University of Sydney, NSW 2006, Australia

Full list of author information is available at the end of the article
}

initiating pathogenic events underlying $\mathrm{AD}$ are still debated, there is strong evidence to suggest that oxidative stress and mitochondrial dysfunction have important roles in the neurodegenerative cascade [3-5]. Therefore, it has been proposed that targeting mitochondrial dysfunction could prove valuable for AD therapeutics [6].

One safe, simple yet effective approach to the repair of damaged mitochondria is photobiomodulation with near-infrared light (NIr). This treatment, which involves the irradiation of tissue with low intensity light in the red to near-infrared wavelength range (600 to $1000 \mathrm{~nm}$ ), was originally pioneered for the healing of superficial wounds [7] but has been recently shown to have efficacy in protecting the central nervous system. While the mechanism of action remains to be elucidated, there is

\section{Ciomed Central}

(c) 2014 Purushothuman et al.; licensee BioMed Central Ltd. This is an Open Access article distributed under the terms of the Creative Commons Attribution License (http://creativecommons.org/licenses/by/2.0), which permits unrestricted use, distribution, and reproduction in any medium, provided the original work is properly cited. 
evidence that NIr preserves and restores cellular function by reversing dysfunctional mitochondrial cytochrome $c$ oxidase (COX) activity, thereby mitigating the production of reactive oxygen species and restoring ATP production to normal levels $[8,9]$.

To date, NIr treatment has yielded neuroprotective outcomes in animal models of retinal damage $[9,10]$, traumatic brain injury [11,12], Parkinson's disease [13-15] and AD [16,17]. Furthermore, NIr therapy has yielded beneficial outcomes in clinical trials of human patients with mild to moderate stroke [18] and depression [19]. This treatment represents a promising alternative to drug therapy because it is safe, easy to apply and has no known side-effects at levels even higher than optimal doses [20].

The aim of this study was to assess the efficacy of NIr in mitigating the brain pathology and associated cellular damage that characterise $\mathrm{AD}$. We utilised two mouse models, each manifesting distinct AD-related pathologies: the K3 tau transgenic model, which develops NFTs $[21,22]$; and the APP/PS1 transgenic model, which develops amyloid plaques [23]. Here, we present histochemical evidence that NIr treatment over a period of 1 month reduces the severity of AD-related pathology and oxidative stress and restores mitochondrial function in brain regions susceptible to neurodegeneration in $A D$, specifically the neocortex and hippocampus. The findings extend our previous NIr work in models of acute neurodegeneration $[13,14]$ to demonstrate that NIr is also effective in protecting the brain against chronic insults due to $\mathrm{AD}$-related genetic aberrations, a pathogenic mechanism that is likely to more closely model the human neurodegenerative condition.

\section{Methods}

\section{Mouse models}

The K3 transgenic mouse model, originally generated as a model of frontotemporal dementia [21,22], harbours a human tau gene with the pathogenic K369I mutation; expression is driven by the neuron-specific mThy1.2 promoter. This model manifests high levels of hyperphosphorylated tau and NFTs by 2 to 3 months of age and cognitive deficits by about 4 months of age [21,22]. We commenced our experiments on $\mathrm{K} 3$ mice and matched $\mathrm{C} 57 \mathrm{BL} / 6$ wildtype (WT) controls at 5 months of age, when significant neuropathology is already present.

The APPswe/PSEN1dE9 (APP/PS1) transgenic mouse model, obtained from the Jackson Laboratory (Stock number 004462; Bar Harbor, ME, USA), harbours two human transgenes: the amyloid beta precursor protein gene (APP) containing the Swedish mutation; and the presenilin-1 gene (PS1) containing a deletion of exon 9 [23]. The APP/ PS1 mice exhibit increased A $\beta$ and amyloid plaques by 4 months of age [24] and cognitive deficits by 6 months of age [25]. We commenced our experiments on APP/PS1 mice and matched $\mathrm{C} 57 \mathrm{BL} / 6 \times \mathrm{C} 3 \mathrm{H}$ WT controls at 7 months of age, when numerous amyloid plaques and associated cognitive deficits are present.

Genotyping of mice was achieved by extracting DNA from tail tips through a modified version of the Hot Shot preparation method [26] and amplifying the transgene sequence by polymerase chain reaction. As reported previously, K3 mice were identified using the primers 5'-GGGTGTCTCCAATGCCTGCTTCTTCAG-3' (forward) and 5'-AAGTCACCCAGCAGGGAGGTGC TCAG-3' (reverse) [21,22] and APP/PS1 mice were genotyped using primers 5' -AGGACTGACCACTCGACCAG-3' (forward) and 5'-CGGGGGTCTAGTTCTGC AT-3' (reverse) [23].

\section{Experimental design}

For each series of experiments on K3 mice (aged 5 months) or APP/PS1 mice (aged 7 months) there were three experimental groups: untreated WT mice, untreated transgenic mice and NIr-treated transgenic mice $(n=5$ mice per experimental group for the K3 series, 15 mice in total; $n=6$ mice per experimental group for the APP/ PS1 series, 18 mice in total). Our design did not include a WT control group exposed to NIr because NIr has no detectable impact on the survival and function of cells in normal healthy brain [13-15]. Given the consistency of the previous results, use of animals for this extra control group did not seem justified [27].

Mice in the NIr-treated groups were exposed to one 90-second cycle of NIr (670 nm) from a light-emitting device (LED) (WARP 10; Quantum Devices, Barneveld, WI, USA) for 5 days per week over 4 consecutive weeks. Light energy emitted from the LED during each 90second treatment equates to $4 \mathrm{Joule} / \mathrm{cm}^{2}$; a total of 80 Joule $/ \mathrm{cm}^{2}$ was delivered to the skull over the 4 weeks. Our measurements of NIr penetration across the fur and skull of a C57BL/6 mouse indicate that $\sim 2.5 \%$ of transmitted light reaches the cortex.

For each treatment, the mouse was restrained by hand and the LED was held 1 to $2 \mathrm{~cm}$ above the head. The LED light generated no heat and reliable delivery of the radiation was achieved [13-15]. For the sham-treated WT, K3 and APP/PS1 groups, animals were restrained in the same way and the device was held over the head, but the light was not switched on. This treatment regime is similar to that used in previous studies where beneficial changes to neuropathology and behavioural signs were reported [13-15].

Experimental animals were housed two or more to a cage and kept in a 12-hour light (<5 lux)/dark cycle at $22^{\circ} \mathrm{C}$; food pellets and water were available ad libitum. All protocols were approved by the Animal Ethics Committee of the University of Sydney. 


\section{Histology and immunohistochemistry}

At the end of the experimental period, mice were anaesthetised by intraperitoneal injection of sodium pentobarbital $(60 \mathrm{mg} / \mathrm{kg})$ and perfused transcardially with $4 \%$ buffered paraformaldehyde. Brains were post fixed for 3 hours, washed with phosphate-buffered saline and cryoprotected in 30\% sucrose/phosphate-buffered saline. Tissue was embedded in OCT compound (ProSciTech, Thuringowa, QLD, Australia) and coronal sections of the neocortex and the hippocampus (between bregma -1.8 and -2.1 ) were cut at $20 \mu \mathrm{m}$ thickness on a Leica cryostat (Nussloch, Germany).

\section{Immunohistochemistry}

For most antibodies, antigen retrieval was achieved using sodium citrate buffer with $0.1 \%$ Triton. Sections were blocked in $10 \%$ normal goat serum and then incubated overnight at $4^{\circ} \mathrm{C}$ with a mouse monoclonal antibody paired helical filaments-tau AT8, 1:500 (Innogenetics, Ghent, Belgium); 4-hydroxynonenal (4-HNE), 1:200 (JaICA, Fukuroi, Shizuoka, Japan); 8-hydroxy-2'-deoxyguanosine (8-OHDG), 1:200 (JaICA); COX, 1:200 (MitoSciences, Eugene, OR, USA) - and/or a rabbit polyclonal antibody (200 kDa neurofilament, 1:500; Sigma, St. Louis, MO, USA). Sections were then incubated for 3 hours at room temperature in Alexa Fluor-488 (green) and/or Alexa Fluor-594 (red) tagged secondary antibodies specific to host species of the primary antibodies (1:1,000; Molecular Probes, Carlsbad, CA, USA). Sections were then counterstained for nuclear DNA with bisbenzimide (Sigma).

Two different but complementary antibodies were used to label $A \beta$ peptide: $6 \mathrm{E} 10$, which recognises residues 1 to 16; and 4G8, which recognises residues 17 to 24 . We have previously used these two antibodies in combination to validate $A \beta$ labelling, demonstrating identical labelling patterns in the rat neocortex and hippocampus [28]. For double labelling using 6E10 antibodies (1:500; Covance, Princeton, NJ, USA) and anti-glial fibrillary acidic protein antibodies (1:1,000; DAKO, Glostrup, Denmark), antigen retrieval was achieved by incubation in $90 \%$ formic acid for 10 minutes, and primary antibody incubation was carried out overnight at room temperature. For labelling using the 4G8 (1:500; Covance) antibody, slides were treated with 3\% $\mathrm{H}_{2} \mathrm{O}_{2}$ in $50 \%$ methanol, incubated in $90 \%$ formic acid and then washed several times in $\mathrm{dH}_{2} \mathrm{O}$ before the blocking step, as described previously [28]. After blocking, sections were incubated overnight at room temperature with 4G8 antibody. Sections were then incubated in biotinylated goat anti-mouse IgG for 1 hour followed by ExtrAvidin peroxidase for 2.5 hours. The sections were then washed and developed with 3,3'-Diaminobenzidine.

Negative control sections were processed in the same fashion as described above except that primary antibodies were omitted. These control sections were immunonegative. Fluorescent images were taken using a Zeiss
Apotome 2, Carl Zeiss, Oberkochen, Germany. Brightfield images were taken using a Nikon Eclipse E800, Nikon Instruments, Melville, NY, USA.

\section{Histology}

NFTs were assessed using the Bielschowsky silver staining method, as described previously [21,22]. Briefly, sections were placed in prewarmed 10\% silver nitrate solution for 15 minutes, washed and then placed in ammonium silver nitrate solution at $40^{\circ} \mathrm{C}$ for a further 30 minutes. Sections were subsequently developed for 1 minute and then transferred to $1 \%$ ammonium hydroxide solution for 1 minute to stop the reaction. Sections were then washed in $\mathrm{dH}_{2} \mathrm{O}$, placed in 5\% sodium thiosulphate solution for 5 minutes, washed, cleared and mounted in dibutyl phathalate xylene.

As described previously [28], $A \beta$ plaques were studied by staining with Congo red, a histological dye that binds preferentially to compacted amyloid with a $\beta$-sheet secondary structure [29]. Briefly, sections were treated with 2.9 $\mathrm{M}$ sodium chloride in $0.01 \mathrm{M} \mathrm{NaOH}$ for 20 minutes and were subsequently stained in filtered alkaline $0.2 \%$ Congo red solution for 1 hour.

\section{Morphological analysis \\ Staining intensity and area measurements}

To quantify the average intensity and area of antibody labelling within the neocortex and hippocampal regions, an integrated morphology analysis was undertaken using MetaMorph software. For each section, the level of nonspecific staining (using an adjacent region of unstained midbrain) was adjusted to a set level to ensure a standard background across different groups. Next, outlines of retrosplenial cortex area 29 and hippocampal CA1 region were traced and the average intensity and area of immunostaining were calculated by the program. Measurements were conducted on $\geq 4$ representative sections per animal and $\geq 3$ animals per experimental group. Statistical analyses were performed in Prism 5.0 (Graphpad, La Jolla, CA, USA) using one-way analysis of variance with Tukey's multiple comparison post test. All values are given as mean \pm standard error of mean.

\section{Amyloid-beta plaque measurements}

Digital brightfield images of 4G8 staining in the neocortical and hippocampal regions (between bregma -1.8 and -2.1 ) were taken at $4 \times$ magnification and analysed with Metamorph, Molecular Devices LLC, Sunnyvale, CA, USA. The software was programmed to measure the number of plaques and the average size of plaques after thresholding for colour. The percentage of area covered by plaques (plaque burden) was calculated by multiplying the number of plaques by the average size of plaques, divided by the area of interest, as described previously [30]. The average number of Congo red-positive plaques in the APP/PS1 brain 
regions was estimated using the optical fractionator method (StereoInvestigator; MBF Science, Williston, VT, USA), as outlined previously [14]. Briefly, systematic random sampling of sites was undertaken using an unbiased counting frame $(100 \mu \mathrm{m} \times 100 \mu \mathrm{m})$. All plaques that came into focus within the frame were counted. Measurements were conducted on $\geq 4$ representative sections per animal and $\geq 3$ animals per experimental group. Plaque numbers and size were analysed using a two-tailed unpaired $t$ test (when variances were equal) or Welch's $t$ test (when variances were unequal). All values are given as mean \pm standard error of mean. For all analyses, investigators were blinded to the experimental groups.

\section{Results}

Evidence of NIr-induced neuroprotection is presented from the neocortex (retrosplenial area) and the hippocampus (CA1 and subiculum), two cortical regions affected in the early stages of human AD [2].
Near-infrared light mitigates the tau pathology of K3 cortex Hyperphosphorylation of the neuronal microtubule stabilising protein tau and the resulting NFTs are much studied features of dementia pathology [2,31]. The K3 mouse model manifests hyperphosphorylated tau and NFTs by 2 to 3 months of age and cognitive deficits by about 4 months of age [21,22]. We observe strong labelling for hyperphosphorylated tau in the neocortex and the hippocampus at 6 months of age; expression appears to plateau after this age, with similar labelling observed in 12-month-old mice (Figure 1A,B,C,D,E,F).

In the retrosplenial area of the neocortex there was a significant overall difference in AT8 immunolabelling for tau between the experimental groups, both when considering average intensity of labelling $(P<0.01$ by analysis of variance; Figure $2 \mathrm{~A})$ and labelled area $(P<0.01$; Figure 2B). Tukey post hoc testing revealed significant differences between the untreated K3 group and the other two groups; labelling was much stronger and more widespread in K3 mice than WT controls (17-fold higher

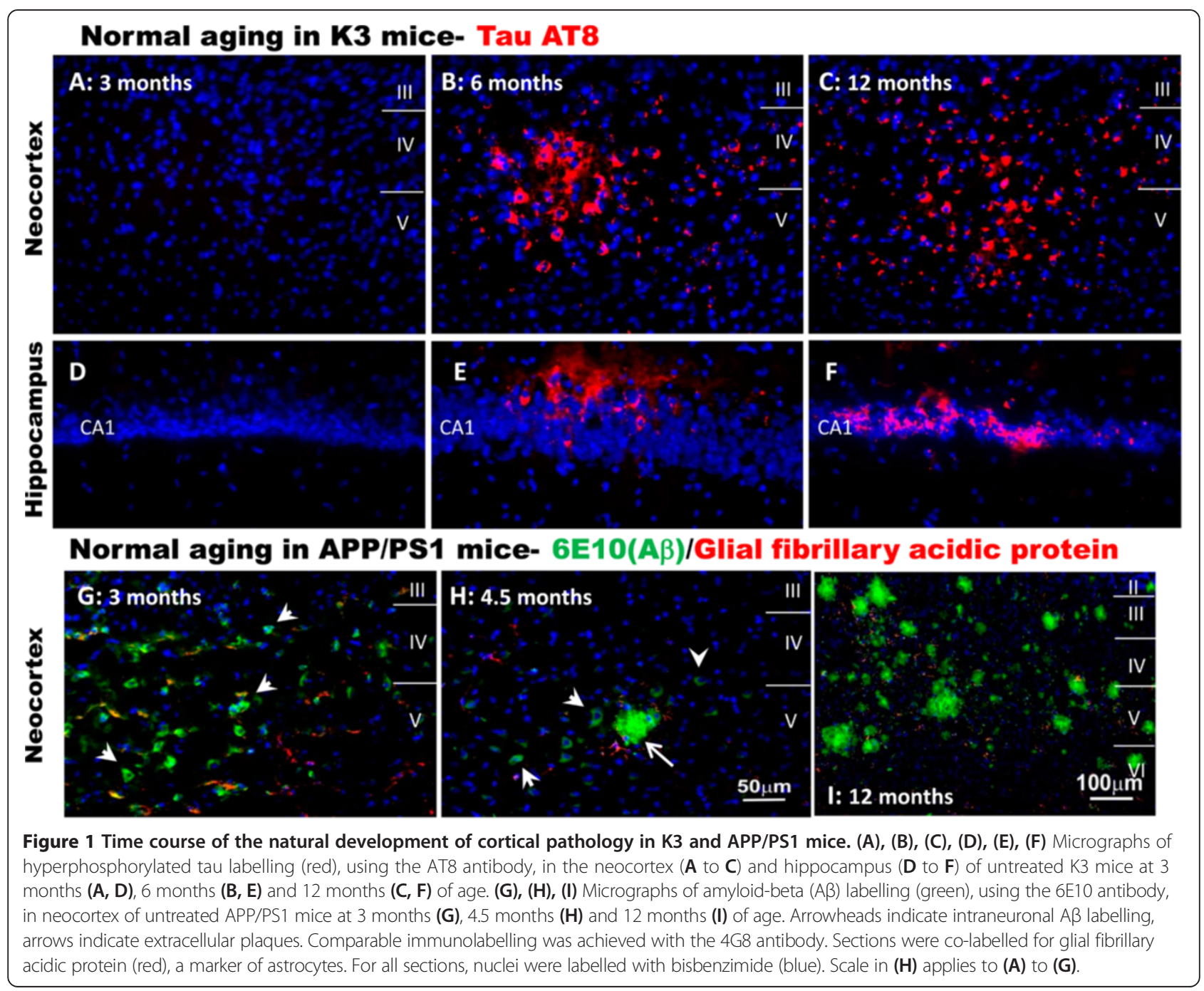




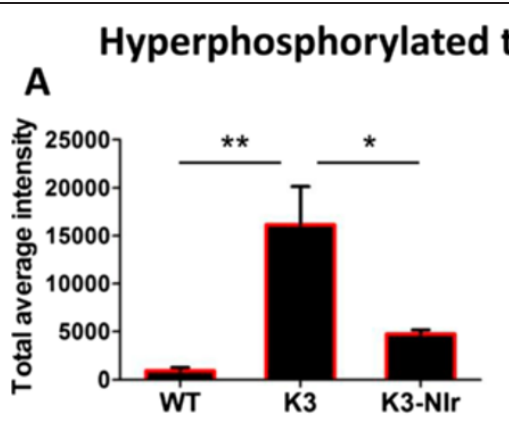

Silver stain
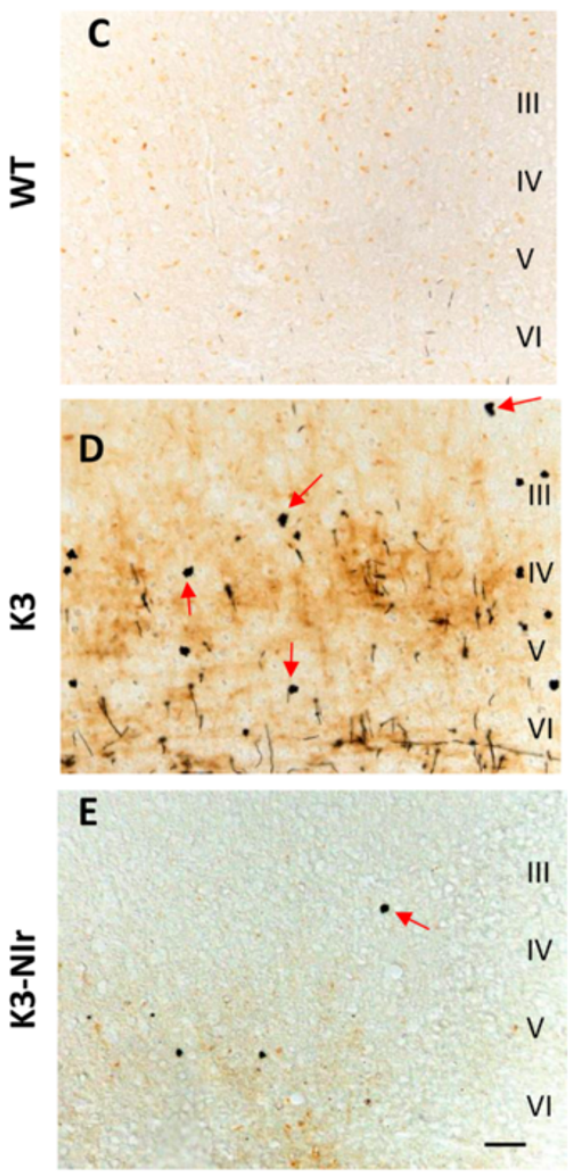

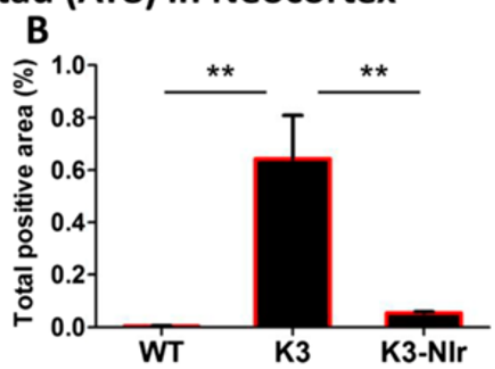

Tau AT8
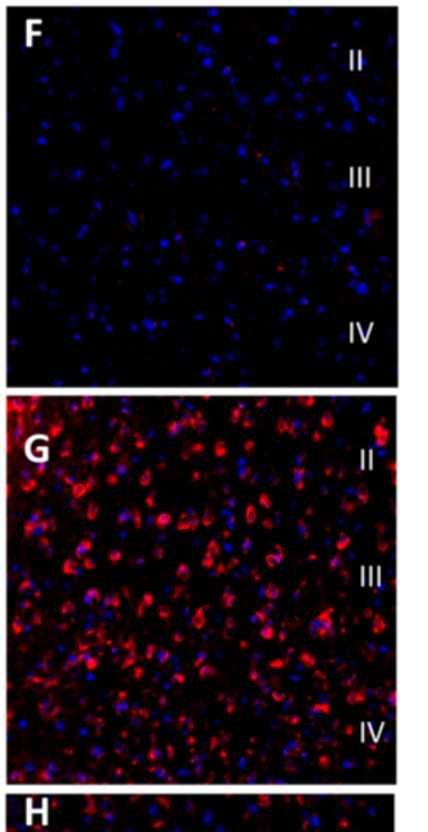

II

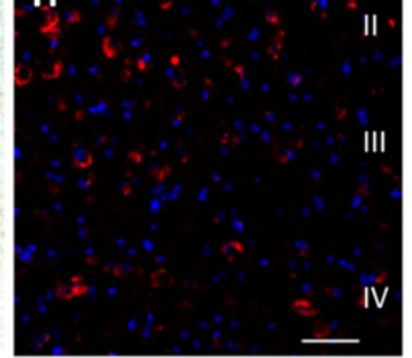

Figure 2 Effect of near-infrared light treatment on hyperphosphorylated tau and neurofibrillary tangles in the neocortex of K3 mice. (A), (B) Quantification of tau AT8 immunolabelling, based on average labelling intensity (A) and labelled area (B). All error bars indicate standard error of mean. ${ }^{*} P<0.05,{ }^{*} P<0.01$. (C), (D), (E) Representative photomicrographs of sections stained with Bielschowsky silver stain to demonstrate neurofibrillary tangles (NFTs). Arrows indicate axonal swellings and NFTs. (F), (G), (H) Representative micrographs of AT8 (red) labelling within neurons of the neocortex retrosplenial area. Nuclei were labelled with bisbenzimide (blue). Scale bars =50 $\mu \mathrm{m}$; scale in (E) applies to (C) and (D), scale in (H) applies to (F) and (G). Nlr, near-infrared light; WT, wildtype.

intensity, $P<0.01$ ), and this labelling was reduced by over $70 \%$ in NIr-treated mice $(P<0.05)$. Interestingly, there was no significant difference between the WT and K3-NIr groups, suggesting that NIr treatment had reduced hyperphosphorylated tau to control levels in K3 mice. A similar trend was observed when considering the NFT pathology (Figure 2C,D,E). In contrast to WT brain, which showed no NFT-like lesions (Figure 2C), the K3 brain contained many ovoid shaped NFT-like lesions (that is, spheroids; Figure 2D). Such structures were less frequent in the K3-NIr brain (Figure 2E).

Similar effects were observed in the hippocampus (Figure 3). There was a significant overall difference between the experimental groups in AT8 immunolabelling 


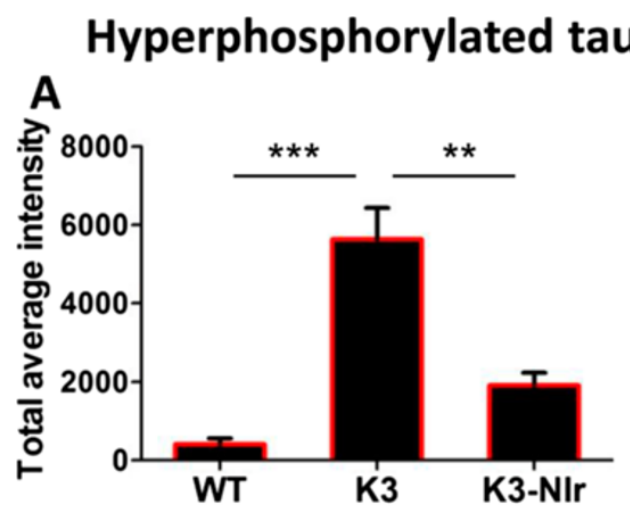

Silver stain in subiculum
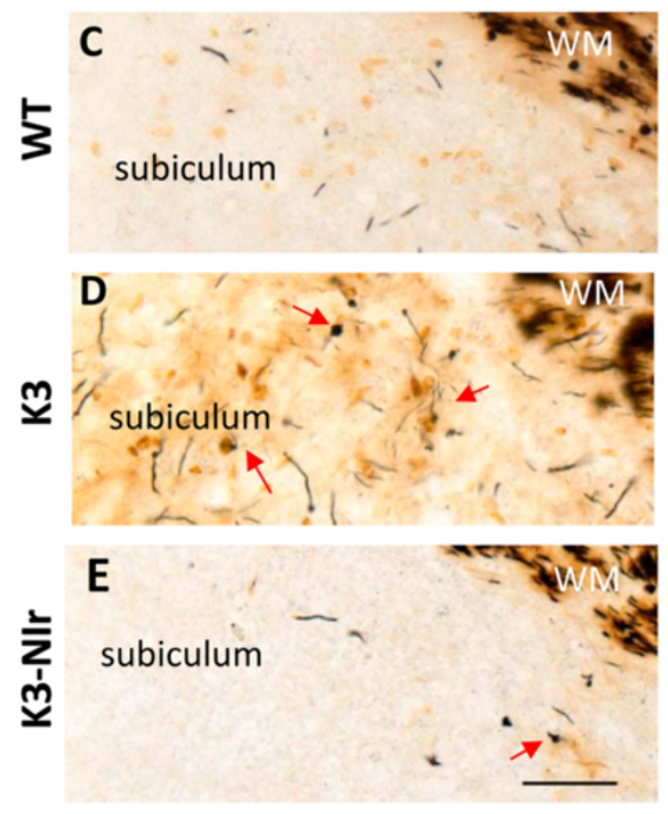

B

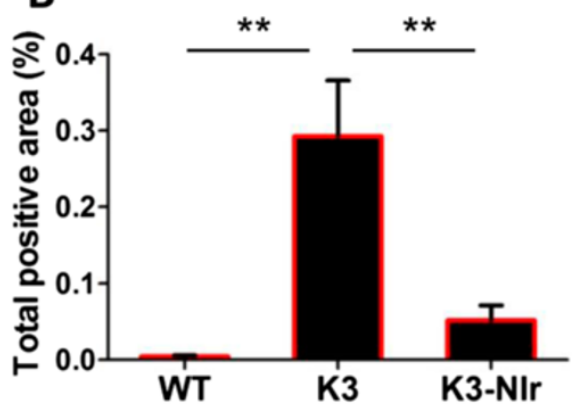

Tau AT8 in CA1
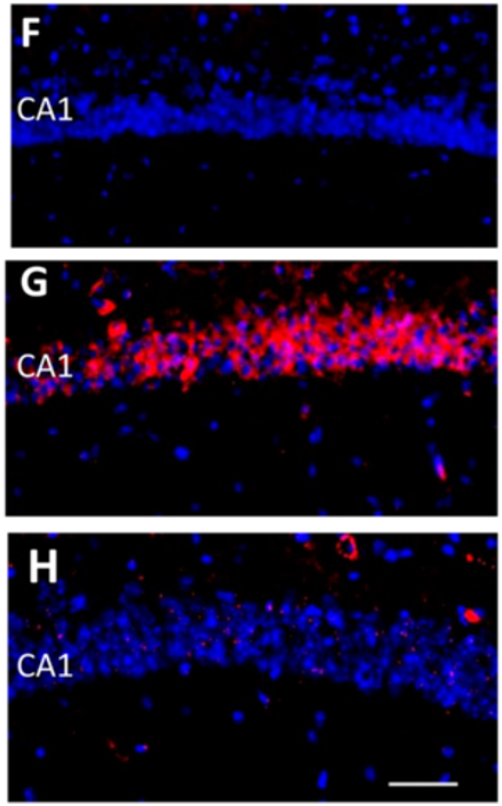

Figure 3 Effect of near-infrared light treatment on hyperphosphorylated tau and neurofibrillary tangles in the hippocampus of K3 mice. (A), (B) Quantification of tau AT8 immunolabelling, based on average labelling intensity (A) and labelled area (B). All error bars indicate standard error of mean. ${ }^{*} P<0.01,{ }^{* * *} P<0.001$. (C), (D), (E) Representative photomicrographs of sections of the hippocampal subiculum area, stained with Bielschowsky silver stain to demonstrate neurofibrillary tangles (NFTs). Arrows indicate axonal swellings and NFTs. The classical silver stain also labelled axons in the white matter core (WM). (F), (G), (H) Representative micrographs of AT8 (red) labelling within hippocampal CA1 pyramidal neurons. Nuclei were labelled with bisbenzimide (blue). Scale bars $=50 \mu \mathrm{m}$; scale in (E) applies to (C) and (D), scale in (H) applies to (F) and (G). Nlr, near-infrared light; WT, wildtype.

of the CA1 pyramidal cells $(P<0.01)$. As for the neocortex, K3 mice showed far greater labelling than WT mice $(17$-fold higher intensity, $P<0.01)$ and this was reduced over $65 \%$ by NIr treatment $(P<0.01)$. Again, there were no significant differences between the WT and K3-NIr groups $(P>0.05)$. Bielschowsky silver staining of the subiculum (Figure 3C,D,E) revealed axonal swellings and spheroids in the hippocampal region of $\mathrm{K} 3$ mice (Figure 3D), which were less pronounced in mice from the K3-NIr group (Figure 3E). No pathology was observed in the hippocampus of WT mice (Figure 3C).
One should note that the large white matter pathways associated with the hippocampus were labelled intensely by silver staining in all three groups (Figure 3C,D,E). This labelling has been described previously and is not associated with any neuropathology [32].

Near-infrared light reduces oxidative stress in K3 cortex Oxidative stress and damage are common features of neurodegenerative diseases such as $\mathrm{AD}$, and may be a precursor to neuronal death [3-5]. We assessed two common markers of oxidative stress: 4-HNE, a toxic end-product of lipid peroxidation that may bind to 

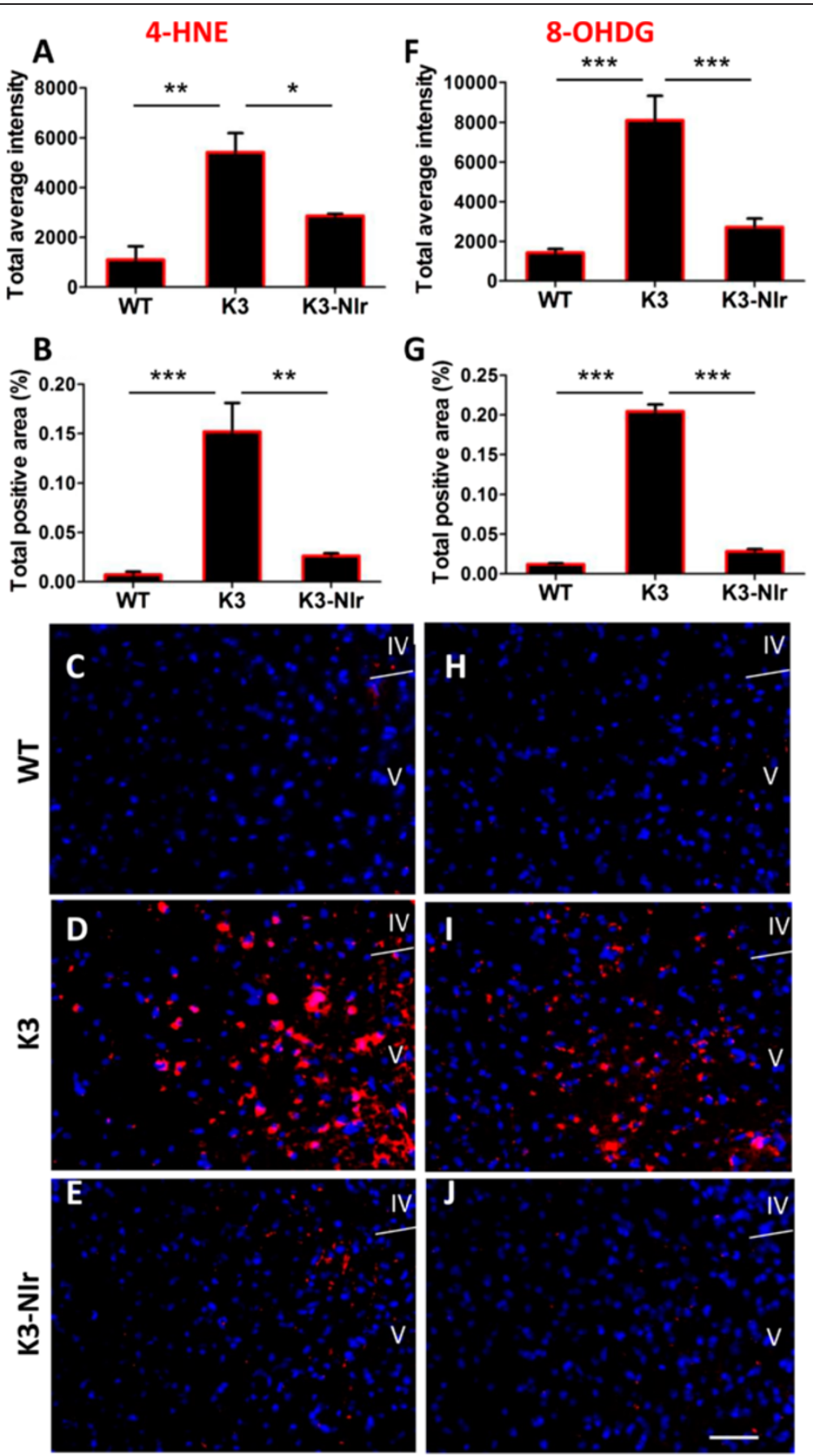

Figure 4 Effect of near-infrared light treatment on oxidative stress markers in the neocortex of K3 mice. (A), (B), (F), (G) Quantification of immunolabelling of two oxidative stress markers, 4-hydroxynonenal (4-HNE; A, B) and 8-hydroxy-2'-deoxyguanosine (8-OHDG; F, G), based on average labelling intensity (A, F) and labelled area (B, G). All error bars indicate standard error of the mean. ${ }^{*} P<0.05,{ }^{* *} P<0.01,{ }^{* * *} P<0.001 .(\mathbf{C})$, (D), (E), (H), (I), (J) Representative micrographs of 4-HNE (red) labelling (C, D, E) and 8-OHDG (red) labelling (H, I, J) within layers IV and V of the neocortical retrosplenial area. Nuclei were labelled with bisbenzimide (blue). Scale bar $=50 \mu \mathrm{m}$; scale in (J) applies to all other micrographs. Nir, near-infrared light; WT, wildtype.

proteins that then trigger mitochondrial dysfunction and cellular apoptosis in AD [33]; and 8-OHDG, a marker for nuclear and mitochondrial DNA oxidation, which is elevated in AD brains [34].
Overall, 4-HNE immunoreactivity in the neocortex was significantly different between the experimental groups (Figure 4 ), by both average labelling intensity $(P<0.01)$ and labelled area $(P<0.001)$. As with AT8 
labelling above, the $\mathrm{K} 3$ group showed a much higher average 4-HNE labelling intensity and area than the WT group (fivefold and 20-fold, respectively) and this labelling was significantly reduced (by $50 \%$ and $80 \%$, respectively) in the K3-NIr group. Again, these measures showed no significant differences between the WT and K3-NIr groups $(P>0.05)$.

Similar patterns were observed for 8-OHDG immunoreactivity. Overall, there was a significant difference between the groups for 8-OHDG immunolabelling, by both average intensity $(P<0.0001)$ and labelled area $(P<0.0001)$. Again the K3 group showed significantly higher 8-OHDG labelling intensity and area than the WT group (sixfold and 17-fold, respectively), and the 8-OHDG labelling intensity and area were significantly reduced in the K3-NIr group relative to untreated K3 (65\% and 85\% reduction, respectively). The intensity and area of 8-OHDG labelling did not differ significantly between the WT and the K3-NIr groups $(P>0.05)$, suggesting that NIr treatment reduces markers of oxidative stress to control levels. The representative photomicrographs of 8-OHDG immunoreactivity in the retrosplenial area (Figure $4 \mathrm{H}, \mathrm{I}, \mathrm{J})$ reflect the quantitative data, with many 8 - $\mathrm{OHDG}^{+}$structures in the $\mathrm{K} 3$ group (Figure 4I) but not in the WT and K3-NIr groups (Figure 4H,J).

\section{Near-infrared light mitigates mitochondrial dysfunction in K3 cortex}

We assessed expression patterns of the mitochondrial enzyme COX in the neocortex and the hippocampus as a marker of mitochondrial function. Overall, there were statistically significant differences in the patterns of COX immunoreactivity between the different experimental groups, both in the neocortex and the hippocampus (both $P<0.0001$; Figure 5). Relative to WT mice, the COX labelling intensity and area were reduced in $\mathrm{K} 3$ mice in both the neocortex and the hippocampus ( $>70 \%$ and $>75 \%$ reductions, respectively). The K3-NIr mice showed a significant recovery of $\mathrm{COX}$ immunoreactivity relative to untreated $\mathrm{K} 3$ mice in both the neocortex ( $>1.7$-fold increase, $P<0.05)$ and the hippocampus $(>3.4$-fold increase, $P<0.001)$. However, recovery was not complete, with K3-NIr mice having significantly lower COX immunoreactivity than WT mice in the neocortex $(\sim 50 \%$, $P<0.001)$ and significantly lower COX labelling intensity $(\sim 20 \%, P<0.05)$ in the hippocampus. These two groups did not differ significantly in COX labelling area in the hippocampus $(P>0.05)$.

\section{Near-infrared mitigates amyloid pathology in APP/PS1 cortex}

Along with NFTs, $A \beta$ plaques are considered a primary pathological hallmark of $\mathrm{AD}$ and $\mathrm{A} \beta$ load is often used as a marker of $\mathrm{AD}$ severity $[1,35]$. We assessed the distribution of $A \beta$ plaques and more immature forms of the $A \beta$ peptide in the neocortex and hippocampus of APP/PS1 mice aged 7 months; this age is after the first signs of intracellular $A \beta$ within cells (at 3 months; Figure 1G) and extracellular $A \beta$ plaques (at 4.5 and 12 months; Figure $1 \mathrm{H}$ and $1 \mathrm{I}$, respectively).

Three quantitative measures of plaque pathology were used: percentage plaque burden, average plaque size and number of plaques. Immunohistochemical labelling with the anti-A $\beta$ antibody 4 G8 revealed a significant reduction in percentage plaque burden (Figure 6A,D), average plaque size (Figure 6B,E) and number of plaques (Figure 6C,F) in both the neocortex and the hippocampus of NIr-treated APP/PS1 mice relative to untreated APP/PS1 controls. Percentage plaque burden was reduced by over $40 \%$ in the neocortex (Figure 6A; $P<0.001$ ) and over $70 \%$ in the hippocampus (Figure 6D; $P<0.01$ ), average plaque size was reduced $25 \%$ in the neocortex (Figure $6 \mathrm{~B}$ ) and $30 \%$ in the hippocampus (Figure 6E), and the number of plaques was reduced by over $20 \%$ in the neocortex (Figure $6 \mathrm{C}$ ) and by over 55\% in the hippocampus (Figure 6F; all $P<0.05$ ).

The photomicrographs of the 4G8 immunoreactivity in Figure 6 reflect the quantitative data described earlier. The WT brain is free of plaques (Figure $6 \mathrm{H}, \mathrm{K}$ ); many $4 \mathrm{Gr}^{+}$plaques (arrows) are present in the neocortex (Figure 6I) and the hippocampus (Figure 6L) of untreated APP/PS1 mice, and fewer plaques are present in NIr-treated APP/PS1 mice (Figure 6J,M). Comparable immunolabelling was achieved using the $6 \mathrm{E} 10$ anti- $\mathrm{A} \beta$ antibody (data not shown).

A similar but less pronounced trend was observed when staining with Congo red (Figure 7), which stains only mature plaques. Mean counts of plaques in the neocortex (Figure 7A) and the hippocampus (Figure 7B) of NIrtreated APP/PS1 brains were lower than mean counts in untreated APP/PS1 brains (reductions $>30 \%$ ). However, the differences did not reach statistical significance; given the findings described above with the 4G8 and 6E10 anti$A \beta$ antibodies, this suggests that NIr may have greatest effect on recently formed $A \beta$ deposits. The micrographs in Figure 7 show that mature plaques were absent from the WT brain (Figure 7C,D) but were present in the neocortex (Figure 7E) and hippocampus (Figure 7F) of untreated APP/PS1 brains. There appeared to be fewer plaques in the NIr-treated APP/PS1 brains (Figure 7G,H).

\section{Discussion}

Using two mouse models with distinct AD-related pathologies (tau pathology in K3, amyloid pathology in APP/ PS1), we report evidence that NIr treatment can mitigate the pathology characteristic of $\mathrm{AD}$ as well as reduce oxidative stress and restore mitochondrial function in brain regions affected early in the disease. Further, the extent of mitigation - to levels less than at the start of treatment suggests that NIr can reverse some elements of ADrelated pathology. 


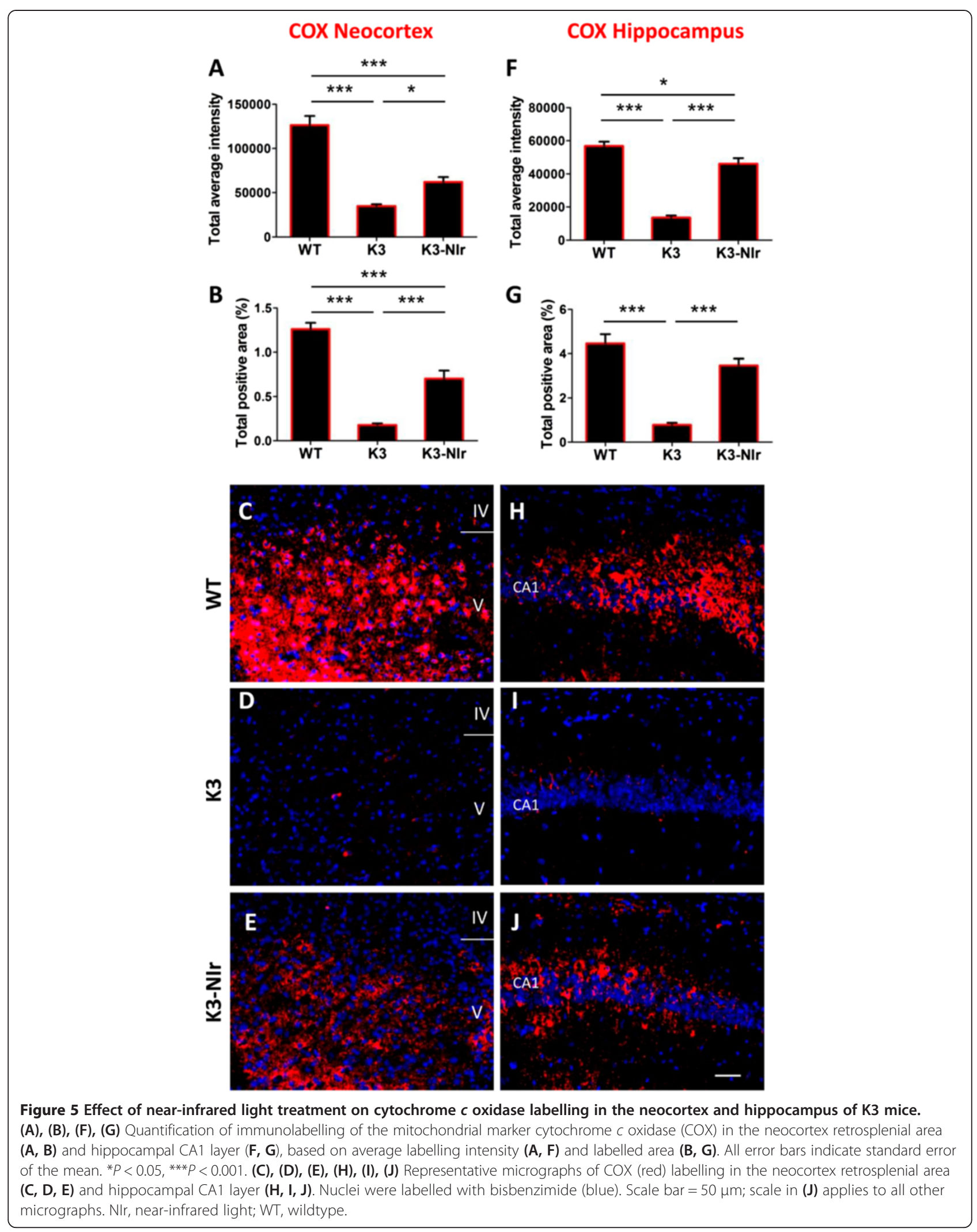




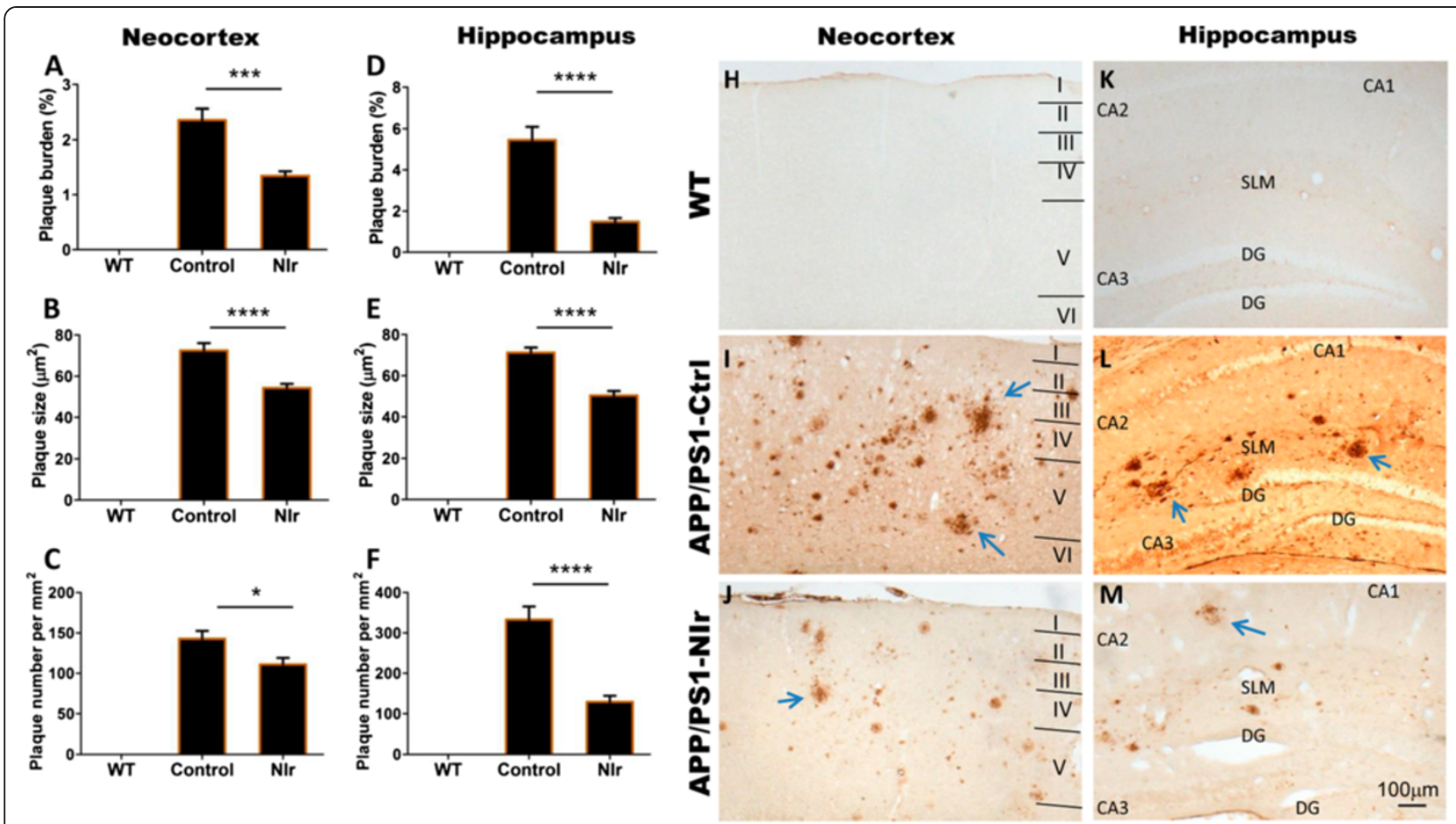

Figure 6 Effect of near-infrared light on amyloid-beta and plaque pathology in APP/PS1 mice. (A), (B), (C), (D), (E), (F) Quantification of amyloid-beta (Aß) 4G8 immunolabelling of amyloid plaques in the neocortex (A, B, C) and hippocampus (D, E, F), based on plaque burden (A, D), plaque size (B, E) and number of plaques (C, F). All error bars indicate standard error of the mean. ${ }^{*} P<0.05,{ }^{* * *} P<0.001$, ${ }^{* * * *} P<0.0001 .(\mathbf{H}),(\mathbf{I}),(\mathbf{J}),(\mathbf{K})$, (L), (M) Representative micrographs showing A labelling with the 4G8 antibody (brown) in the neocortex (H, I, J) and hippocampus (K, L, M). Arrows indicate plaques. Scale bar = $100 \mu \mathrm{m}$; scale in (M) applies to all other micrographs. DG, dentate gyrus of hippocampus; NIr, near-infrared light; SLM, stratum lacunosum moleculare; WT, wildtype.

The present results add to our previous findings of NIrinduced neuroprotection in models of toxin-induced acute neurodegeneration (that is, MPTP-induced parkinsonism). When incorporated into the growing body of evidence that NIr can also protect against CNS damage in models of stroke, traumatic brain injury and retinal degeneration [9-12,36], the findings provide a basis for trialling NIr treatment as a strategy for protection against neurodegeneration from a range of causes. Present evidence is based on the use of multiple methods, immunohistochemical and histological, to demonstrate pathological features (for example, $4 \mathrm{G} 8$ antibody labelling and Congo red staining for amyloid plaques, AT8 antibody labelling and Bielschowsky silver staining for NFTs).

\section{Relationship to previous studies}

The present study focused on pathological features considered characteristic of AD, as well as on signs of cellular damage (for example, oxidative stress, mitochondrial dysfunction) that have been demonstrated in AD and in animal models [2-4]. Our observations in the K3 strain add to previous studies by providing the first evidence in this strain of extensive oxidative damage and mitochondrial dysfunction [27].
Our findings are consistent with previous reports of the effects of red to infrared light on AD pathology in animal models. De Taboada and colleagues assessed the capacity of $808 \mathrm{~nm}$ laser-sourced infrared radiation, delivered three times per week over 6 months, to reduce pathology in an APP transgenic model of A $\beta$ amyloidosis [17]. Treatment led to a reduction in plaque number, amyloid load and inflammatory markers, an increase in ATP levels and mitochondrial function, and mitigation of behavioural deficits. De Taboada and colleagues commenced treatment at 3 months of age, before the expected onset of amyloid pathology and cognitive effects. Similarly, Grillo and colleagues reported that 1,072 nm infrared light, applied 4 days per week for 5 months, reduces AD-related pathology in another APP/PS1 transgenic mouse model (TASTPM) [16]. These investigators also initiated light treatment before the onset of pathology, at 2 months of age. Both studies thus provide evidence that infrared radiation can slow the progression of cerebral degeneration in these models. The present results confirm these observations, in two distinct transgenic strains; they also confirm that the wound-healing and neuroprotective effects of red-infrared length do not vary qualitatively with wavelength, over a wide range. 

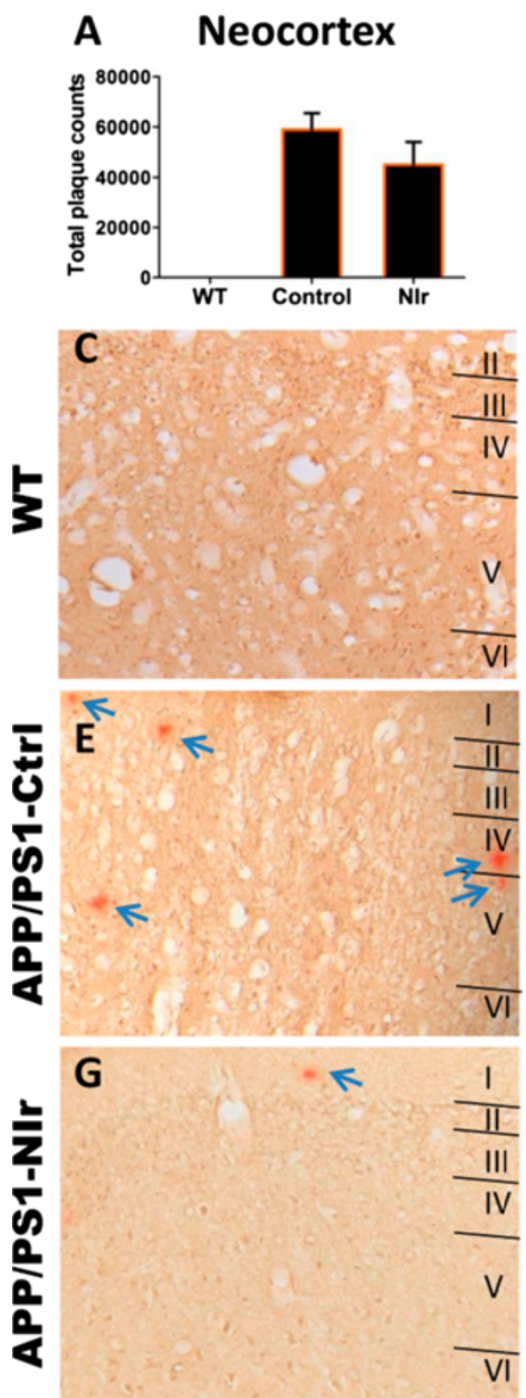

\section{B Hippocampus}
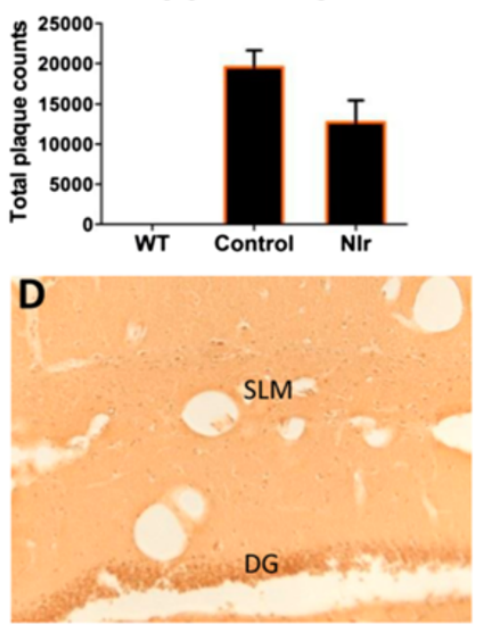

$\mathbf{F}$
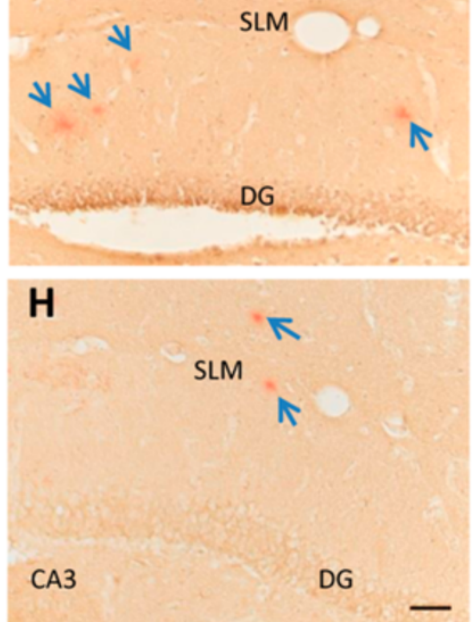

Figure 7 Effect of near-infrared light on Congo red-positive plaque numbers in APP/PS1 mice. (A), (B) Quantification of Congo red-positive plaque counts in the neocortex (A) and hippocampus (B). All error bars indicate standard error of the mean. (C), (D), (E), (F), (G), (H) Representative micrographs showing Congo red staining of plaques in the neocortex $(\mathbf{C}, \mathbf{E}, \mathbf{G})$ and hippocampus $(\mathbf{D}, \mathbf{F}, \mathbf{H})$. Arrows indicate plaques. Scale bar $=50 \mu \mathrm{m}$; scale in (H) applies to all other micrographs. DG, dentate gyrus of hippocampus; NIr, near-infrared light; SLM, stratum lacunosum moleculare; WT, wildtype.

\section{Evidence of reversal of pathology}

Previous reports have described the natural history of the K3 [21,22] and APP/PS1 transgenic models [24,37]. Based on these previous reports and our own baseline data (Figure 1), significant brain pathology and functional deficits are present in both models at the ages when we commenced treatment. Our results therefore suggest that significant reversal of pathology has been induced by the NIr treatment. This has implications for clinical practice, where most patients are not diagnosed until pathogenic mechanisms have already been initiated and resultant neurologic symptoms manifest [15,27].

This evidence that AD-related neuropathology can be transient - appear then disappear - is not novel. Garcia-
Alloza and colleagues described evidence of the transient deposition of $A \beta$, including the formation of plaque-like structures, in a transgenic model of $A \beta$ deposition [24]. Reversal of such pathology, by interventions such as NIr treatment, may therefore be possible. However our results suggest that reversal may also be limited to recently formed, immature plaques, as we observed a significant NIr-induced reduction in immunolabelling with the 4G8 and $6 \mathrm{E} 10$ antibodies but no significant difference in Congo red staining. Because the 4G8 and 6E10 antibodies recognise various forms of $A \beta$, while Congo red stains only mature, compacted plaques, a reasonable deduction is that NIr treatment reduces only the transient, recently formed $A \beta$ deposits, with no substantial effect on mature plaques. 
As there is still no consensus as to the pathogenic roles of different forms of $A \beta$, it is unclear how this might impact on the therapeutic potential of NIr in a clinical setting.

\section{Mechanisms}

The mechanisms underlying the neuroprotective actions of red to infrared light are not completely understood. There is considerable evidence that NIr photobiomodulation enhances mitochondrial function and ATP synthesis by activating photoacceptors such as $\mathrm{COX}$ and increasing electron transfer in the respiratory chain, while also reducing harmful reactive oxygen species [38-40]. NIr photobiomodulation could also upregulate protective factors such as nerve growth factor and vascular endothelial growth factor [41,42] and mesenchymal stem cells [43] that could target specific areas of degeneration.

The ability of NIr to reduce the expression of hyperphosphorylated tau, which in turn reduces oxidative stress [44], may be key to its neuroprotective effect. Oxidative stress and free radicals increase the severity of cerebrovascular lesions $[45,46]$, mitochondrial dysfunction [4,47], oligomerisation of $A \beta[5,48]$ and tauopathies and cell death $[48,49]$ in AD. Considering the brain's high consumption of oxygen and consequent susceptibility to oxidative stress, mitigating such stressors would probably have a pronounced protective effect [50].

\section{Conclusions}

Overall, our results in two transgenic mouse models with existing AD-related pathology suggest that lowenergy NIr treatment can reduce characteristic pathology, oxidative stress and mitochondrial dysfunction in susceptible regions of the brain. These results, when taken together with those in other models of neurodegeneration, strengthen the notion that NIr is a viable neuroprotective treatment for a range of neurodegenerative conditions. We believe this growing body of work provides the impetus to begin trialling NIr treatment as a broadbased therapy for $\mathrm{AD}$ and other neurodegenerations.

\section{Abbreviations}

A $\mathrm{B}$ : Amyloid-beta; AD: Alzheimer's disease; APP: Amyloid beta precursor protein gene; COX: Cytochrome c oxidase; 4-HNE: 4-hydroxynonenal; LED: Light-emitting diode; NFT: Neurofibrillary tangle; NIr: Near-infrared light; 8-OHDG: 8-hydroxy-2'-deoxyguanosine; PS1: Presenilin 1; WT: Wildtype.

\section{Competing interests}

The authors declare that they have no competing interests.

\section{Authors' contributions}

SP undertook the bulk of the experimental work and analysis and wrote the manuscript. DMJ and JM were involved with the analysis of the data and the writing of the manuscript. CN was involved with genotyping and treating the animals. JS was involved in conceiving and designing the study and the writing of the manuscript. All authors read and approved the final manuscript.

\section{Acknowledgments}

The authors thank Tenix Corporation, Sir Zelman Cowen Universities Fund and Bluesand Foundation for funding. They are grateful to Prof. Lars Ittner for providing the breeding litter for K369l mice, and to Dr Louise Cole and the Bosch Advanced Microscopy facility for the help with MetaMorph. Sharon Spana was splendid for her technical help. DMJ is supported by a National Health and Medical Research Council of Australia (NHMRC) Early Career Fellowship.

\section{Author details}

'Bosch Institute, University of Sydney, NSW 2006, Australia. ${ }^{2}$ Discipline of Physiology, Anderson Stuart Building F13, University of Sydney, NSW 2006, Australia. ${ }^{3}$ Discipline of Anatomy \& Histology, Anderson Stuart Building F13, University of Sydney, NSW 2006, Australia.

Received: 11 September 2013 Accepted: 3 December 2013

Published: 3 January 2014

\section{References}

1. Hardy J, Selkoe DJ: The amyloid hypothesis of Alzheimer's disease: progress and problems on the road to therapeutics. Science 2002, 297:353-356.

2. Braak H, Braak E: Staging of Alzheimer's disease-related neurofibrillary changes. Neurobiol Aging 1995, 16:271-278.

3. Nunomura A, Perry G, Aliev G, Hirai K, Takeda A, Balraj EK, Jones PK, Ghanbari H, Wataya T, Shimohama S, Chiba S, Atwood CS, Petersen RB, Smith MA: Oxidative damage is the earliest event in Alzheimer disease. I Neuropathol Exp Neurol 2001, 60:759-767.

4. Yao J, Irwin RW, Zhao L, Nilsen J, Hamilton RT, Brinton RD: Mitochondrial bioenergetic deficit precedes Alzheimer's pathology in female mouse model of Alzheimer's disease. Proc Natl Acad Sci USA 2009, 106:14670-14675

5. Stone J: What initiates the formation of senile plaques? The origin of Alzheimer-like dementias in capillary haemorrhages. Med Hypotheses 2008, 71:347-359.

6. Calabrese V, Guagliano E, Sapienza M, Panebianco M, Calafato S, Puleo E, Pennisi G, Mancuso C, Butterfield DA, Stella AG: Redox regulation of cellular stress response in aging and neurodegenerative disorders: role of vitagenes. Neurochem Res 2007, 32:757-773.

7. Whelan HT, Smits RL Jr, Buchman EV, Whelan NT, Turner SG, Margolis DA, Cevenini V, Stinson H, Ignatius R, Martin T, Martin T, Cwiklinski J, Philippi AF, Graf WR, Hodgson B, Gould L, Kane M, Chen G, Caviness J: Effect of NASA light-emitting diode irradiation on wound healing. J Clin Laser Med Surg 2001, 19:305-314.

8. Desmet KD, Paz DA, Corry JJ, Eells JT, Wong-Riley MT, Henry MM, Buchmann EV, Connelly MP, Dovi JV, Liang HL, Henshel DS, Yeager RL, Millsap DS, Lim J, Gould LJ, Das R, Jett M, Hodgson BD, Margolis D, Whelan HT: Clinical and experimental applications of NIR-LED photobiomodulation. Photomed Laser Surg 2006, 24:121-128.

9. Eells JT, Wong-Riley MT, VerHoeve J, Henry M, Buchman EV, Kane MP, Gould LJ, Das R, Jett M, Hodgson BD, Margolis D, Whelan HT: Mitochondrial signal transduction in accelerated wound and retinal healing by near-infrared light therapy. Mitochondrion 2004, 4:559-567.

10. Natoli R, Zhu Y, Valter K, Bisti S, Eells J, Stone J: Gene and noncoding RNA regulation underlying photoreceptor protection: microarray study of dietary antioxidant saffron and photobiomodulation in rat retina. Mol Vis 2010, 16:1801-1822.

11. Xuan W, Vatansever F, Huang L, Wu Q, Xuan Y, Dai T, Ando T, Xu T, Huang YY, Hamblin MR: Transcranial low-level laser therapy improves neurological performance in traumatic brain injury in mice: effect of treatment repetition regimen. PLoS One 2013, 8:e53454.

12. Oron A, Oron U, Streeter J, de Taboada L, Alexandrovich A, Trembovler V, Shohami E: Low-level laser therapy applied transcranially to mice following traumatic brain injury significantly reduces long-term neurological deficits. J Neurotrauma 2007, 24:651-656.

13. Moro C, Torres N, El Massri N, Ratel D, Johnstone DM, Stone J, Mitrofanis J, Benabid AL: Photobiomodulation preserves behaviour and midbrain dopaminergic cells from MPTP toxicity: evidence from two mouse strains. BMC Neurosci 2013, 14:40

14. Shaw VE, Spana S, Ashkan K, Benabid AL, Stone J, Baker GE, Mitrofanis J: Neuroprotection of midbrain dopaminergic cells in MPTP-treated mice after near-infrared light treatment. J Comp Neurol 2010, 518:25-40.

15. Peoples C, Spana S, Ashkan K, Benabid AL, Stone J, Baker GE, Mitrofanis J: Photobiomodulation enhances nigral dopaminergic cell survival in a 
chronic MPTP mouse model of Parkinson's disease. Parkinsonism Relat Disord 2012, 18:469-476.

16. Grillo SL, Duggett NA, Ennaceur A, Chazot PL: Non-invasive infra-red therapy $(1072 \mathrm{~nm})$ reduces beta-amyloid protein levels in the brain of an Alzheimer's disease mouse model, TASTPM. J Photochem Photobiol B 2013, 123:13-22.

17. De Taboada L, Yu J, El-Amouri S, Gattoni-Celli S, Richieri S, McCarthy T, Streeter J, Kindy MS: Transcranial laser therapy attenuates amyloid-beta peptide neuropathology in amyloid-beta protein precursor transgenic mice. J Alzheimers Dis 2011, 23:521-535.

18. Lampl Y, Zivin JA, Fisher M, Lew R, Welin L, Dahlof B, Borenstein P, Andersson B, Perez J, Caparo C, llic S, Oron U: Infrared laser therapy for ischemic stroke: a new treatment strategy: results of the NeuroThera Effectiveness and Safety Trial-1 (NEST-1). Stroke 2007, 38:1843-1849.

19. Schiffer F, Johnston AL, Ravichandran C, Polcari A, Teicher MH, Webb RH, Hamblin MR: Psychological benefits 2 and 4 weeks after a single treatment with near infrared light to the forehead: a pilot study of 10 patients with major depression and anxiety. Behav Brain Funct 2009, 5:46.

20. Tuby H, Hertzberg E, Maltz L, Oron U: Long-term safety of low-level laser therapy at different power densities and single or multiple applications to the bone marrow in mice. Photomed Laser Surg 2013, 31:269-273.

21. Ittner LM, Fath T, Ke YD, Bi M, van Eersel J, Li KM, Gunning P, Gotz J: Parkinsonism and impaired axonal transport in a mouse model of frontotemporal dementia. Proc Natl Acad Sci USA 2008, 105:15997-16002.

22. van Eersel J, Ke YD, Liu X, Delerue F, Kril JJ, Gotz J, Ittner LM: Sodium selenate mitigates tau pathology, neurodegeneration, and functional deficits in Alzheimer's disease models. Proc Natl Acad Sci USA 2010, 107:13888-13893.

23. Jankowsky JL, Fadale DJ, Anderson J, Xu GM, Gonzales V, Jenkins NA, Copeland NG, Lee MK, Younkin LH, Wagner SL, Younkin SG, Borchelt DR: Mutant presenilins specifically elevate the levels of the 42 residue beta-amyloid peptide in vivo: evidence for augmentation of a 42-specific gamma secretase. Hum Mol Genet 2004, 13:159-170.

24. Garcia-Alloza M, Robbins EM, Zhang-Nunes SX, Purcell SM, Betensky RA, Raju S, Prada C, Greenberg SM, Bacskai BJ, Frosch MP: Characterization of amyloid deposition in the APPswe/PS1dE9 mouse model of Alzheimer disease. Neurobiol Dis 2006, 24:516-524.

25. Cao D, Lu H, Lewis TL, Li L: Intake of sucrose-sweetened water induces insulin resistance and exacerbates memory deficits and amyloidosis in a transgenic mouse model of Alzheimer disease. J Biol Chem 2007, 282:36275-36282

26. Truett GE, Heeger P, Mynatt RL, Truett AA, Walker JA, Warman ML: Preparation of PCR-quality mouse genomic DNA with hot sodium hydroxide and tris (HotSHOT). Biotechniques 2000, 29:52-54.

27. Purushothuman S, Nandasena C, Johnstone DM, Stone J, Mitrofanis J: The impact of near-infrared light on dopaminergic cell survival in a transgenic mouse model of parkinsonism. Brain Res 2013, 1535C:61-70.

28. Purushothuman S, Marotte L, Stowe S, Johnstone DM, Stone J: The response of cerebral cortex to haemorrhagic damage: experimental evidence from a penetrating injury model. PLoS One 2013, 8:e59740.

29. Wilcock DM, Gordon MN, Morgan D: Quantification of cerebral amyloid angiopathy and parenchymal amyloid plaques with Congo red histochemical stain. Nat Protoc 2006, 1:1591-1595.

30. Yan Q, Zhang J, Liu H, Babu-Khan S, Vassar R, Biere AL, Citron M, Landreth $\mathrm{G}$ : Anti-inflammatory drug therapy alters beta-amyloid processing and deposition in an animal model of Alzheimer's disease. J Neurosci 2003, 23:7504-7509

31. Augustinack JC, Schneider A, Mandelkow EM, Hyman BT: Specific tau phosphorylation sites correlate with severity of neuronal cytopathology in Alzheimer's disease. Acta Neuropathol 2002, 103:26-35.

32. Bruck W, Bitsch A, Kolenda H, Bruck Y, Stiefel M, Lassmann H: Inflammatory central nervous system demyelination: correlation of magnetic resonance imaging findings with lesion pathology. Ann Neurol 1997, 42:783-793.

33. Sayre LM, Zelasko DA, Harris PL, Perry G, Salomon RG, Smith MA 4-Hydroxynonenal-derived advanced lipid peroxidation end products are increased in Alzheimer's disease. J Neurochem 1997, 68:2092-2097.

34. Mecocci P, MacGarvey U, Beal MF: Oxidative damage to mitochondrial DNA is increased in Alzheimer's disease. Ann Neurol 1994, 36:747-751.

35. Trinchese F, Liu S, Battaglia F, Walter S, Mathews PM, Arancio O: Progressive age-related development of Alzheimer-like pathology in APP/PS1 mice. Ann Neurol 2004, 55:801-814.
36. Oron A, Oron U, Chen J, Eilam A, Zhang C, Sadeh M, Lampl Y, Streeter J, DeTaboada L, Chopp M: Low-level laser therapy applied transcranially to rats after induction of stroke significantly reduces long-term neurological deficits. Stroke 2006, 37:2620-2624.

37. Blanchard V, Moussaoui S, Czech C, Touchet N, Bonici B, Planche M, Canton T, Jedidi I, Gohin M, Wirths O, Bayer TA, Langui D, Duyckaerts C, Tremp G, Pradier $L$ : Time sequence of maturation of dystrophic neurites associated with $A \beta$ deposits in APP/PS1 transgenic mice. Exp Neurol 2003, 184:247-263.

38. Karu T: Mitochondrial mechanisms of photobiomodulation in context of new data about multiple roles of ATP. Photomed Laser Surg 2010, 28:159-160

39. Wilden L, Karthein R: Import of radiation phenomena of electrons and therapeutic low-level laser in regard to the mitochondrial energy transfer. J Clin Laser Med Surg 1998, 16:159-165.

40. Wong-Riley MT, Bai X, Buchmann E, Whelan HT: Light-emitting diode treatment reverses the effect of TTX on cytochrome oxidase in neurons. Neuroreport 2001, 12:3033-3037.

41. Hou JF, Zhang H, Yuan X, Li J, Wei YJ, Hu SS: In vitro effects of low-level laser irradiation for bone marrow mesenchymal stem cells: proliferation, growth factors secretion and myogenic differentiation. Lasers Surg Med 2008, 40:726-733.

42. Tuby $H$, Maltz L, Oron U: Modulations of VEGF and iNOS in the rat heart by low level laser therapy are associated with cardioprotection and enhanced angiogenesis. Lasers Surg Med 2006, 38:682-688.

43. Tuby H, Maltz L, Oron U: Induction of autologous mesenchymal stem cells in the bone marrow by low-level laser therapy has profound beneficial effects on the infarcted rat heart. Lasers Surg Med 2011, 43:401-409.

44. Stamer K, Vogel R, Thies E, Mandelkow E, Mandelkow EM: Tau blocks traffic of organelles, neurofilaments, and APP vesicles in neurons and enhances oxidative stress. J Cell Biol 2002, 156:1051-1063.

45. Aliev G, Smith MA, Seyidov D, Neal ML, Lamb BT, Nunomura A, Gasimov EK, Vinters HV, Perry G, LaManna JC, Friedland RP: The role of oxidative stress in the pathophysiology of cerebrovascular lesions in Alzheimer's disease. Brain Pathol 2002, 12:21-35.

46. Hamel E, Nicolakakis N, Aboulkassim T, Ongali B, Tong XK: Oxidative stress and cerebrovascular dysfunction in mouse models of Alzheimer's disease. Exp Physiol 2008, 93:116-120.

47. Zhu X, Perry G, Moreira Pl, Aliev G, Cash AD, Hirai K, Smith MA: Mitochondrial abnormalities and oxidative imbalance in Alzheimer disease. J Alzheimers Dis 2006, 9:147-153.

48. Zhang $X$, Le W: Pathological role of hypoxia in Alzheimer's disease. Exp Neurol 2010, 223:299-303.

49. Wen Y, Yang S, Liu R, Brun-Zinkernagel AM, Koulen P, Simpkins JW: Transient cerebral ischemia induces aberrant neuronal cell cycle re-entry and Alzheimer's disease-like tauopathy in female rats. J Biol Chem 2004, 279:22684-22692.

50. Uttara B, Singh AV, Zamboni P, Mahajan RT: Oxidative stress and neurodegenerative diseases: a review of upstream and downstream antioxidant therapeutic options. Curr Neuropharmacol 2009, 7:65-74.

doi:10.1186/alzrt232

Cite this article as: Purushothuman et al:: Photobiomodulation with near infrared light mitigates Alzheimer's disease-related pathology in cerebral cortex - evidence from two transgenic mouse models. Alzheimer's Research \& Therapy $20146: 2$. 